\title{
Social Regulation of Negative Valence Systems During Development
}

\author{
Shannon $\mathrm{Hu}^{\dagger}$, Katherine Packard ${ }^{\dagger}$ and Maya Opendak* \\ Kennedy Krieger Institute, Solomon H. Snyder Department of Neuroscience, The Johns Hopkins University School of \\ Medicine, Baltimore, MD, United States
}

The ability to sense, perceive, and respond appropriately to aversive cues is critical for survival. Conversely, dysfunction in any of these pathway components can lead to heightened avoidance of neutral or rewarding cues, such as social partners. The underlying circuitry mediating both negative valence processing and social behavior is particularly sensitive to early life experience, but mechanisms linking experience to pathology remain elusive. Previous research in humans, rodents, and non-human primates has highlighted the unique neurobiology of the developing infant and the role of the caregiver in mediating the infant's negative valence circuitry, and the importance of this early social relationship for scaffolding lasting social behavior. In this review, we summarize the current literature on the development of negative valence circuits in the infant and their social regulation by the caregiver following both typical and adversityrearing. We focus on clinically-relevant research using infant rodents which highlights the amygdala and its interface with the mesolimbic dopamine system through innervation from the ventral tegmental area (VTA) as a locus of dysfunction following early-life adversity. We then describe how these circuits are recruited to perturb life-long social behavior following adversity and propose additional therapeutic targets in these circuits with an eye toward developing age-appropriate interventions.

Keywords: stress, attachment, mother-infant dyad, amygdala, learning, social behavior, dopamine

\section{INTRODUCTION}

Social support is associated with positive health outcomes, and research has demonstrated that the presence of a social-support figure can reduce psychological and physiological responses to aversive experiences (Inagaki and Eisenberger, 2012; Hornstein and Eisenberger, 2017). Indeed, the critical need for social support during adverse experiences has been underscored during the COVID-19 pandemic, in which isolated individuals have faced increased adverse mental health outcomes (Sahi et al., 2021). In order for this social support to be effective, the brain-body systems responding to aversive cues, or negative valence circuits, must be functioning appropriately. In a highly dynamic environment, the ability to sense, perceive, and respond appropriately to aversive cues is critical for survival. Conversely, dysfunction in any of these pathway components can lead to impaired threat responses and is a key impairment in disorders such as PTSD and anxiety (Callaghan et al., 2019; Meyer and Lee, 2019; Abend et al., 2020).

Impaired negative valence systems can be damaging not only when an individual fails to avoid a threat, but also if the individual fails to approach potential rewards due 
to heightened threat responses. As an example, for most species, access to food, protection, and receptive mates requires a motivation to approach social partners, whereas failure to do so prevents access to these key resources. Furthermore, failing to engage in social approach behavior can prevent access to social support during adversity. As noted above, this can lead to increased susceptibility to highly aversive stimuli, chronic stress system engagement, and further maladaptive remodeling of negative valence systems.

The plasticity of these circuits that renders them open to social regulation also renders them vulnerable to perturbation by extreme adverse experiences, such as trauma. Specifically, mounting evidence suggests that these circuits are particularly sensitive to experiences during early development, such as early caregiving adversity (Demers et al., 2018; VanTieghem and Tottenham, 2018; Birnie et al., 2020; Tottenham, 2020). As will be discussed below, species-atypical care can perturb threat responses and undermine the effectiveness of social partners during aversive cues later in life. In this way, the unique impact of social stimuli is two-fold-both scaffolding these developing negative circuits and regulating their function in adulthood. Although these circuits are a critical locus of dysfunction in psychopathology, we have limited understanding of the mechanisms linking early social experience, negative valence circuits, and social behavior. However, recent advances in circuit dissection techniques in infant rodent models have provided much-needed insight into this question.

Here, we review the literature on the development of these circuits, their role in social behavior, and the impact of social experience on their function. We focus on clinically-relevant research using infant rodents which permits circuit dissection techniques to ask, how do these systems develop normally, and how does early trauma impact their lasting function to perturb social behavior? We center our discussion on the early social niche of the altricial infant, the caregiver-infant dyad, as this relationship shows unique impacts on the development of negative valence systems.

\section{Why Focus on the Infant-Caregiver Relationship?}

The role of the social partner in regulating developing negative valence circuits is rooted in the unique relationship between the infant and caregiver across altricial species. In these species, infants are born immature and depend on the caregiver for survival, including the provision of food, shelter, protection, and physiological regulation. John Bowlby's Attachment Theory drew on observations from comparative research that infants across altricial species work to maintain contact with the attachment figure, with evolution and survival placing heavy selection bias on these infant-caregiver dyads (Bowlby, 1965, 1978). Rapid attachment is a key component of the infantcaregiver relationship early in life. An example is imprinting in birds (Hess, 1962), which demonstrates an example of rapid and robust attachment immediately after birth. Similarly, non-human primates attach to caregivers that provide contact comfort, a process that is required for healthy development (Harlow and Harlow, 1965).
Forming attachments also impacts altricial infants' interaction with immediate threats in their environment. When faced with threatening cues, the motorically immature infant is unable to fight or flee and instead seeks protection from the caregiver (Coss and Penkunas, 2016). The caregiver also regulates the infant's neurobehavioral responses to threats-a process termed social buffering, whereby the caregiver acts as a "safe haven" for the young and attenuates the child's fear response to potentially threatening or stressful stimuli (Hennessy et al., 2009; Gunnar et al., 2015; Doom et al., 2017; Hornstein and Eisenberger, 2017). Social buffering has been observed across species, including rodents, non-human primates, and humans.

\section{NEURAL CIRCUITS SUPPORTING INFANT SOCIAL BUFFERING}

The neural circuits supporting social buffering involve suppression of cortisol, or its non-human animal analog corticosterone (CORT), from the hypo-thalamic-pituitary axis during stress (Hennessy et al., 2006; Faustino et al., 2017; Howell et al., 2017). This attenuation in turn impacts multiple brain regions typically engaged during threat processing, particularly the amygdala (Fuzzo et al., 2015; Opendak et al., 2019; RobinsonDrummer et al., 2019). The activation of the amygdala is greatly attenuated in human children during a mildly stressful task when the caregiver is present (Gunnar and Donzella, 2002; Gee et al., 2014). Additionally, the mechanisms underlying the recruitment of the amygdala in threat acquisition, consolidation, and expression are believed to be well-conserved across mammalian species (Maren and Fanselow, 1996; Fanselow and LeDoux, 1999; Phelps and LeDoux, 2005; Johansen et al., 2011).

Rodent research has helped elucidate how and when this brain region becomes involved in threat processing, and the impact of caregivers on this process. In typically developing infant rats, the amygdala is not functionally engaged in threat learning during the first few days of life; manipulations to inhibit activity in the amygdala have no effect prior to postnatal (PN) day 10 (Raineki et al., 2009). As a result, these infant rats, or pups, do not form conditioned aversions to stimuli with negative valence, though they are capable of experiencing physical pain and forming amygdala-independent aversions (Rudy and Cheatle, 1983; Blass, 1997; Barr, 2011). This early amygdala inactivity, combined with the unique functioning of the infant odor processing circuitry (anterior piriform cortex, olfactory bulb, and locus coeruleus), means that pups learn to approach any cue associated with the caregiver, even if that cue is aversive (Sullivan et al., 1992, 2000; Sullivan and Wilson, 1994).

Gradual engagement of the amygdala in threat processing occurs alongside developmental changes in endogenous CORT levels (Thompson et al., 2008). In early life, plasma CORT levels are typically very low in rats, a phenomenon termed the stress hyporesponsive period (SHRP; Levine et al., 1985, 1988; Levine, 2001; Hennessy et al., 2006). The gradual rise in CORT as the pup ages has been found to be causal in inducing plasticity in the amygdala and conferring the ability to learn threat: exogenous systemic injection of CORT at PN8 prematurely recruits the amygdala in threat learning processes while adrenalectomy in 


\section{Adversity-rearing}
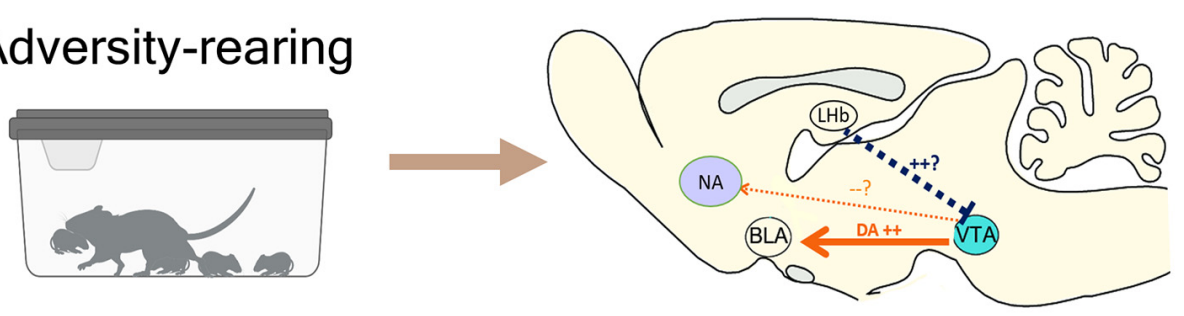

Adversity-reared Rat

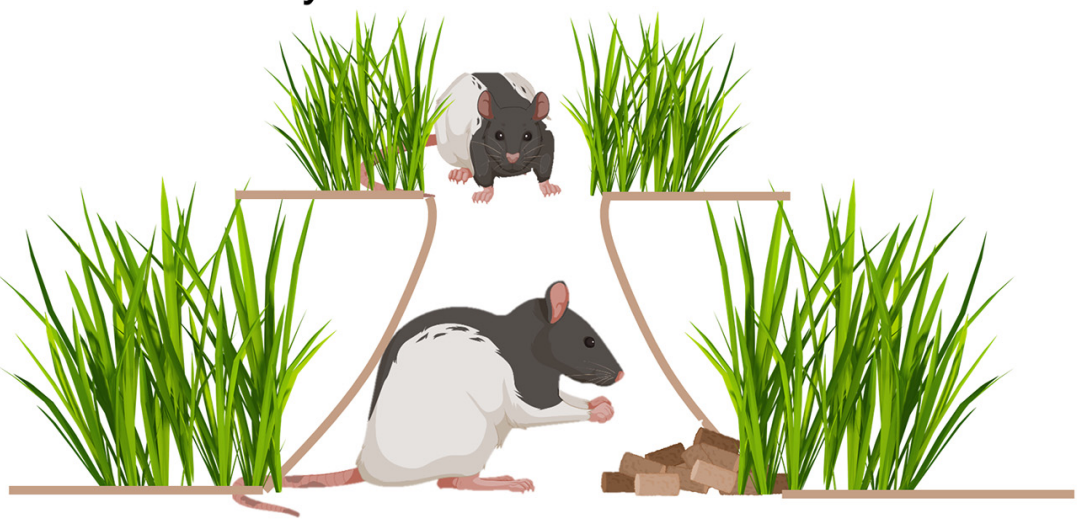

\section{Social Partner}

FIGURE 1 | Remodeled negative valence circuits inhibit social behavior after adversity. Following early life adversity with a caregiver, increased dopaminergic innervation from the ventral tegmental area (VTA) to the basolateral amygdala (BLA) results in decreased social approach toward a novel peer. Measurements during the adversity itself show that whereas caregiver presence typically suppresses dopamine (DA) release in the BLA during threat exposure, repeated adversity with the caregiver is accompanied by enhanced amygdala reactivity and DA release in the caregiver's presence. This suggests that negative valence circuits are engaged in processing the caregiver and this produces lasting hyperactivity of these circuits in responding to social partners. Research now turns to the lateral habenula ( $\mathrm{LH} \mathrm{b}$ ) as the site of dysfunction in social behavior, as this region inhibits DA release from the VTA to the classical reward locus of the nucleus accumbens. Based on recent work, we propose a model whereby early adversity produces LHb hyperactivity (blue dashed line) and inhibited DA release to the accumbens (NA); combined with enhanced DA release in the VTA, these circuit changes decrease the rewarding salience of social partners.

PN12 rats prevents threat expression and amygdala activation (Upton and Sullivan, 2010). The molecular mechanism of this plasticity has also been traced to the trafficking of GluA1 and GluA2 AMPA receptor subunits to synapses in the basolateral amygdala (BLA) and activation of upstream protein kinases protein kinase $\mathrm{M}$ zeta $(\mathrm{PKM} \zeta)$ and iota/lambda $(\mathrm{PKCl} / \lambda)$ in response to elevated CORT. The increased GluA1/2 ratio stabilizes synapses in the amygdala and is required for the formation of threat memories (Huganir and Nicoll, 2013; Oliver et al., 2016; Opendak et al., 2018).

Endogenous CORT levels increase around PN10, an age at which rat pups begin to wander away from the nest and nibble solid food (Sullivan and Holman, 2010; Upton and Sullivan, 2010). This change is accompanied by a transition from the sensitive period for attachment formation to a period when amygdala-dependent threat learning can occur to promote avoidant responses to threat-predicting cues. A similar transition occurs in humans when fear of strangers emerges in young children (Callaghan et al., 2019). One key feature of this developmental window is the new ability of the caregiver to regulate the pup's stress response, whereby the presence of the caregiver or caregiver odor robustly decreases amygdala activity and plasma corticosterone levels, and actively promotes attachment behavior (Moriceau and Sullivan, 2006). 
The caregiver's presence switches what is learned about the stimulus-specifically, caregiver presence during conditioning switches learned aversions to learned preferences during this period, a striking reversal which has recently been documented in children (Tottenham et al., 2019). From PN16 onwards in rats, the power of the caregiver or social partner transitions to a more modulatory function, similar to the effect of social partners in adulthood (Eisenberger et al., 2011; Hornstein and Eisenberger, 2017). A similar shift has been observed in human children, where the caregiver has the power to regulate amygdala-PFC connectivity in younger children but not adolescents (Gee et al., 2014).

Caregiver regulation of infant threat processing affects the midbrain dopamine (DA) circuit involving the amygdala. Decades of microdialysis studies have shown that caregiver inputs such as presence and milk decrease DA release from the ventral tegmental area (VTA), the primary source of dopamine to the amygdala (Tamborski et al., 1990; Andersen et al., 1992; Kehoe et al., 1998). Furthermore, caregiver presence prevents DA release in the BLA during threat (Barr et al., 2009), while DA has been shown to be necessary for BLA long-term plasticity in adults to promote learning (Rosenkranz and Grace, 2002; Lorétan et al., 2004; Kienast et al., 2008; Fadok et al., 2009; Tye et al., 2010; Ng et al., 2018). During the period when the caregiver can toggle preference vs. aversion in rat pups (PN10-15), the caregiver has been shown to regulate the broader mesolimbic dopamine circuit, including the VTA, BLA, and nucleus accumbens (Opendak et al., 2019). As pups develop past weaning (>PN21), the BLA circuit partners impacted by the mother switches from the VTA to the prefrontal cortex (Robinson-Drummer et al., 2019). In post-weaning pups, maternal presence buffers mPFC reactivity to threats, similar to the effect observed in young adults viewing photos of attachment figures during exposure to stressors (Eisenberger et al., 2011). Interestingly, the lateral habenula $(\mathrm{LHb})$, a brain region that shows functional engagement during aversive cues and inhibits DA release from the VTA, continues to be impacted by caregiver presence even in older PN28 pups (Packard et al., 2021).

Taken together, these data suggest that infant negative valence systems have a unique function. Instead of processing threatening or aversive cues to generate avoidance responses, these circuits interpret the caregiver as the ultimate arbiter of ambiguous cues. As a result, the caregiver has immense control over the infant's emotional environment at an age when these brain circuits are still developing.

\section{EARLY LIFE ADVERSITY DEGRADES SOCIAL REGULATION OF NEGATIVE VALENCE CIRCUITS}

As noted above, the adaptive features of approaching the caregiver ensure that attachments are formed, regardless of the quality of the care received. However, abusive and/or neglectful care can impact the quality of attachment that is formed and in turn, the ability of the caregiver to regulate the infant. For example, caregivers in stressful or neglectful rearing conditions have impaired ability to decrease infant stress responses in the Strange Situation Procedure, a canonical laboratory procedure measuring attachment quality in children (Ainsworth and Bell, 1970; Nachmias et al., 1996). The quality of attachment can also be evaluated by the robustness of the child's oscillatory responses to caregiver cues, as measured with EEG (Stamoulis et al., 2015; Perone and Gartstein, 2019; Pratt et al., 2019). Children reared in adverse caregiving conditions also show decreased fMRI response in the amygdala and prefrontal cortex to caregiver cues (Callaghan et al., 2019; Tottenham, 2020).

Recent work in rodents has dissected specific features of compromised caregiver buffering of infant negative valence systems. When pups were reared using the ScarcityAdversity paradigm from PN8-12, in which mother rats are given limited bedding and roughly handle pups, we observed degraded neurobehavioral impact of reunion with a caregiver at PN13 following separation in a rodent Strange Situation procedure-similar to effects observed in children. However, we also were able to record cortical oscillations during adversity-rearing itself and observed that within this context, nurturing maternal inputs, such as grooming and milk ejection, failed to produce expected changes in cortical oscillations (Opendak et al., 2020). These results suggest that impaired processing of nurturing cues, rather than adverse care itself, may drive degraded attachment.

Work in rodent pups has also permitted deconstruction of naturalistic adversity conditions to further identify mechanisms linking experience to the outcome. For instance, the mere presence of a caregiver during repeated adversity was sufficient to produce neurobehavioral deficits in pups, including amygdala dysfunction and social behavior impairments observed at PN13. Specifically, these impairments were observed if pups experienced repeated shock or exogenous CORT administration in the presence of an anesthetized mother, but not alone, from PN8-12 (Raineki et al., 2019; Opendak et al., 2021).

We now understand that impaired social buffering involves dopamine release in the amygdala from the VTA. This mesolimbic dopamine-BLA interface appears to integrate the effects of development, experience, and social buffering (Atzil et al., 2017; Opendak et al., 2019). In typically developing pups at PN12-14, caregiver presence during threat buffers VTA, which is disrupted by adversity-rearing (Opendak et al., 2019). Furthermore, microdialysis in pups shows that repeated shock in the presence of the caregiver increases DA in BLA, and results in failure of the caregiver to buffer amygdala plasticity in response to threat (Opendak et al., 2021). Collectively, these findings define the necessary combination of stress and caregiver presence to disrupt caregiver regulation of negative valence circuits and highlight that adverse maternal behavior per se is not necessary to recapitulate the neurobehavioral effects of adversity-rearing.

\section{IMPLICATIONS FOR SOCIAL BEHAVIOR}

Early social regulation of these negative valence circuits, such as the mesolimbic DA-BLA interface, scaffolds function of 
these circuits in social behavior across the lifespan. Crossspecies data suggest that the amygdala function in social behavior shows developmental transitions. In humans, the amygdala is responsive to fearful stimuli as early as 6-9 months of age (Jessen and Grossmann, 2014; Graham et al., 2016), but its role in social behavior is less well understood. In non-human primates, bilateral amygdala lesions impair social behavior in adulthood but not infancy (Bachevalier et al., 2001; Goursaud and Bachevalier, 2007; Raper et al., 2014). Similarly, pharmacological and optogenetic lesion studies in rodents suggest that amygdala engagement is atypical in infant social behavior (Raineki et al., 2019; Opendak et al., 2021). This early lack of amygdala involvement is in line with the unique ecological niche of the infant which promotes an early social behavior bias toward approaching the mother under threat and safety (Fox et al., 2005; Moriceau and Sullivan, 2006; Coss and Penkunas, 2016; Shultz et al., 2018).

Recent circuit dissection work in rodent pups has highlighted the role of dopaminergic projections from the VTA to the BLA in modulating developmentally-appropriate social behavior (see Figure 1; Opendak et al., 2021). Optogenetic inhibition of the BLA and VTA terminals in the BLA increased social approach in older (PN23) but not younger pups (PN14), suggesting these regions are not involved in inhibiting social behavior in young pups. This work also showed that early life adversity can prematurely engage the amygdala to put a brake on the social approach towards the caregiver. More specifically, dopamine release in the BLA is both necessary and sufficient in producing social avoidance behaviors following adversity. Early-life adversity with the mother (both naturalistic Scarcity-Adversity and repeated shock with mom) from PN8-12 increased dopamine in the pup BLA, and its blockade through pharmacological and optogenetic techniques rescued typical social behavior at pre and post-weaning ages. Conversely, optogenetic stimulation of the VTA to BLA circuit in control-reared pups inhibited typical social approach at PN14. Taken together, these data show that dopaminergic innervation of the BLA is atypical during early social behavior and recruitment of this circuit transitions a system biasing social approach toward the caregiver toward one favoring a balance of approach and avoidance as infants mature.

\section{REMODELING OF EXTENDED NEGATIVE VALENCE CIRCUITS}

The VTA-BLA interface does not exist in isolation, and many mesolimbic structures interface with the VTA to promote adaptive social behavior. Research now turns to circuit partners of the VTA and their specific roles in development. One such circuit partner is the lateral habenula, a small, phylogenetically conserved structure in the posterior-dorsalmedial end of the thalamus with rich connections to both prefrontal cortex and midbrain monoaminergic systems (Hu et al., 2020). As mentioned above, this brain region, considered an "anti-reward" center, responds to aversive cues and sends projections to inhibit DA release from the VTA (Proulx et al., 2014; Baker et al., 2015). Hyperactivity in this structure has been consistently implicated in the pathophysiology of depression, suggesting it is likely a target of early trauma (Simmons et al., 2020). Our recent research on this circuit has found that the LHb is engaged in threat processing at a pre-weaning age (PN18) in both typically and adversity-reared pups. However, previous adversityrearing reduced caregiver buffering of the LHb during threat presentation in post-weaning animals (Packard et al., 2021). Early life adversity also significantly alters functional connectivity patterns between the LHb and downstream circuit partners such as the VTA and substantia nigra, producing a more adult-like connectivity profile. The dysregulated LHb connectivity and $\mathrm{LHb}$ hyperactivity of rats experiencing early life stress suggest there may be greater inhibition of downstream targets, such as the VTA, and heightened aversion to potentially threatening stimuli. However, further dissection of specific projections and cell types mediating these differences is needed.

\section{COULD SOME CHANGES TO SOCIAL BEHAVIOR BE ADAPTIVE?}

While many studies are quick to highlight perceived deficits in children experiencing early life adversity, it should be noted that many changes in functioning may reflect an optimized behavioral strategy (Ellis et al., 2017, 2020). Indeed, taking a more avoidant approach following repeated exposure to an aversive social environment may be adaptive in some circumstances. Such optimizations, called "hidden talents," may require specialized testing to uncover and are a fundamental framework to prevent further stigmatization of children raised in adverse environments. Additional examples include individual differences in measures of resilience (Masten et al., 2004; Southwick et al., 2014) and observations of post-traumatic growth (Tedeschi and Calhoun, 2004) following early life adversity across species. This concept of circuit optimization to suit environmental demands has been proposed not only for social behavior circuits but also for others involved in threat processing and reward-seeking (Opendak et al., 2017; Cushman et al., 2021; Hanson et al., 2021).

\section{CONCLUDING REMARKS}

Disrupted social behavior is a core symptom of many psychiatric and developmental disorders, including autism, anxiety, and depression (Crawley, 2004; Miller, 2007; Kennedy and Adolphs, 2012; Patel et al., 2019). The ontogeny of these disorders is poorly understood, but clinical research has identified early adversity as a significant risk factor in the scaffolding of lifelong social deficits (Teicher et al., 2002, 2003; Raineki et al., 2012, 2015; Tottenham, 2012; Gee et al., 2013; Malter Cohen et al., 2013; Hanson et al., 2015). More recent evidence suggests that adversity within the social context of the attachment figure renders the infant uniquely vulnerable (Raineki et al., 
2019; Opendak et al., 2021). Using invasive circuit dissection techniques previously impossible in pups, here we review how the maternal signal becomes compromised during repeated social adversity and highlight how this may impact social behavior through perturbation of negative valence circuits. This work has highlighted the mesolimbic dopamine interface with the amygdala as a site dysfunction in both caregiver regulation of the infant and infant social behavior. Future work targeting this circuit and upstream regions, such as the habenula, will be critical for developing age-appropriate interventions following early-life adversity.

\section{REFERENCES}

Abend, R., Gold, A. L., Britton, J. C., Michalska, K. J., Shechner, T., Sachs, J. F., et al. (2020). Anticipatory threat responding: associations with anxiety, development and brain structure. Biol. Psychiatry 87, 916-925. doi: 10.1016/j.biopsych.2019. 11.006

Ainsworth, M. D., and Bell, S. M. (1970). Attachment, exploration and separation: illustrated by the behavior of one-year-olds in a strange situation. Child Dev. 41, 49-67. doi: 10.2307/1127388

Andersen, S. L., Gazzara, R. A., Robinson, S. R., and Smotherman, W. P. (1992). Effect of milk of dopamine release in the newborn rat: an in vivo microdialysis study. Brain Res. Dev. Brain Res. 68, 286-288. doi: 10.1016/01653806(92)90073-6

Atzil, S., Touroutoglou, A., Rudy, T., Salcedo, S., Feldman, R., Hooker, J. M., et al. (2017). Dopamine in the medial amygdala network mediates human bonding. Proc. Natl. Acad. Sci. U S A 114, 2361-2366. doi: 10.1073/pnas.1612233114

Bachevalier, J., Malkova, L., and Mishkin, M. (2001). Effects of selective neonatal temporal lobe lesions on socioemotional behavior in infant rhesus monkeys (Macaca mulatta). Behav. Neurosci. 115, 545-559. doi: 10.1037//0735-7044.115. 3.545

Baker, P. M., Oh, S. E., Kidder, K. S., and Mizumori, S. J. Y. (2015). Ongoing behavioral state information signaled in the lateral habenula guides choice flexibility in freely moving rats. Front. Behav. Neurosci. 9:295. doi: $10.3389 /$ fnbeh. 2015.00295

Barr, G. A. (2011). Formalin-induced c-fos expression in the brain of infant rats. J. Pain 12, 263-271. doi: 10.1016/j.jpain.2010.09.005

Barr, G. A., Moriceau, S., Shionoya, K., Muzny, K., Gao, P., Wang, S., et al. (2009). Transitions in infant learning are modulated by dopamine in the amygdala. Nat. Neurosci. 12, 1367-1369. doi: 10.1038/nn.2403

Birnie, M. T., Kooiker, C. L., Short, A. K., Bolton, J. L., Chen, Y., and Baram, T. Z. (2020). Plasticity of the reward circuitry after early-life adversity: mechanisms and significance. Biol. Psychiatry 87, 875-884. doi: 10.1016/j.biopsych.2019.12. 018

Blass, E. M. (1997). Interactions between contact and chemosensory mechanisms in pain modulation in 10-day-old rats. Behav. Neurosci. 111, 147-154. doi: 10.1037//0735-7044.111.1.147

Bowlby, J. (1965). Attachment. New York: Basic Books.

Bowlby, J. (1978). Attachment theory and its therapeutic implications. Adolesc. Psychiatry 6, 5-33.

Callaghan, B. L., Gee, D. G., Gabard-Durnam, L., Telzer, E. H., Humphreys, K. L., Goff, B., et al. (2019). Decreased amygdala reactivity to parent cues protects against anxiety following early adversity: an examination across 3 years. Biol. Psychiatry Cogn. Neurosci. Neuroimaging 4, 664-671. doi: 10.1016/j.bpsc.2019. 02.001

Callaghan, B., Meyer, H., Opendak, M., Van Tieghem, M., Harmon, C., Li, A., et al. (2019). Using a developmental ecology framework to align fear neurobiology across species. Annu. Rev. Clin. Psychol. 15, 345-369. doi: 10.1146/annurevclinpsy-050718-095727

Coss, R. G., and Penkunas, M. J. (2016). Sex difference in choice of concealed or exposed refuge sites by preschool children viewing a model leopard in a

\section{AUTHOR CONTRIBUTIONS}

$\mathrm{SH}, \mathrm{KP}$, and $\mathrm{MO}$ wrote the review. All authors contributed to the article and approved the submitted version.

\section{FUNDING}

We would like to thank National Institutes of Health (NIH) for funding of this research (BRAIN R00MH124434) and the Brain and Behavior Research Foundation (Young Investigator Grant to $\mathrm{MO}$ ).

playground simulation of antipredator behavior. Int. J. Psychol. Res. 9, 8-19. doi: $10.21500 / 20112084.2325$

Crawley, J. N. (2004). Designing mouse behavioral tasks relevant to autistic-like behaviors. Ment. Retard. Dev. Disabil. Res. Rev. 10, 248-258. doi: 10.1002/mrdd. 20039

Cushman, J. D., Drew, M. R., and Krasne, F. B. (2021). The environmental sculpting hypothesis of juvenile and adult hippocampal neurogenesis. Prog. Neurobiol. 199:101961. doi: 10.1016/j.pneurobio.2020.101961

Demers, L. A., McKenzie, K. J., Hunt, R. H., Cicchetti, D., Cowell, R. A., Rogosch, F. A., et al. (2018). Separable effects of childhood maltreatment and adult adaptive functioning on amygdala connectivity during emotion processing. Biol. Psychiatry Cogn. Neurosci. Neuroimaging 3, 116-124. doi: 10.1016/j.bpsc.2017.08.010

Doom, J. R., Doyle, C. M., and Gunnar, M. R. (2017). Social stress buffering by friends in childhood and adolescence: effects on HPA and oxytocin activity. Soc. Neurosci. 12, 8-21. doi: 10.1080/17470919.2016.1149095

Eisenberger, N. I., Master, S. L., Inagaki, T. K., Taylor, S. E., Shirinyan, D., Lieberman, M. D., et al. (2011). Attachment figures activate a safety signalrelated neural region and reduce pain experience. Proc. Natl. Acad. Sci. U S A 108, 11721-11726. doi: 10.1073/pnas.1108239108

Ellis, B. J., Abrams, L. S., Masten, A. S., Sternberg, R. J., Tottenham, N., and Frankenhuis, W. E. (2020). Hidden talents in harsh environments. Dev Psychopathol. doi: 10.1017/S0954579420000887. [Online ahead of print].

Ellis, B. J., Bianchi, J., Griskevicius, V., and Frankenhuis, W. E. (2017). Beyond risk and protective factors: an adaptation-based approach to resilience. Perspect. Psychol. Sci. 12, 561-587. doi: 10.1177/1745691617693054

Fadok, J. P., Dickerson, T. M., and Palmiter, R. D. (2009). Dopamine is necessary for cue-dependent fear conditioning. J. Neurosci. 29, 11089-11097. doi: 10.1523/JNEUROSCI.1616-09.2009

Fanselow, M. S., and LeDoux, J. E. (1999). Why we think plasticity underlying Pavlovian fear conditioning occurs in the basolateral amygdala. Neuron 23 229-232. doi: 10.1016/s0896-6273(00)80775-8

Faustino, A. I., Tacao-Monteiro, A., and Oliveira, R. F. (2017). Mechanisms of social buffering of fear in zebrafish. Sci. Rep. 7:44329. doi: 10.1038/srep44329

Fox, N. A., Henderson, H. A., Marshall, P. J., Nichols, K. E., and Ghera, M. M. (2005). Behavioral inhibition: linking biology and behavior within a developmental framework. Annu. Rev. Psychol. 56, 235-262. doi: 10.1146/annurev.psych.55.090902.141532

Fuzzo, F., Matsumoto, J., Kiyokawa, Y., Takeuchi, Y., Ono, T., and Nishijo, H. (2015). Social buffering suppresses fear-associated activation of the lateral amygdala in male rats: behavioral and neurophysiological evidence. Front. Neurosci. 9:99. doi: 10.3389/fnins.2015.00099

Gee, D. G., Gabard-Durnam, L. J., Flannery, J., Goff, B., Humphreys, K. L., Telzer, E. H., et al. (2013). Early developmental emergence of human amygdalaprefrontal connectivity after maternal deprivation. Proc. Natl. Acad. Sci. U S A 110, 15638-15643. doi: 10.1073/pnas.1307893110

Gee, D. G., Gabard-Durnam, L., Telzer, E. H., Humphreys, K. L., Goff, B., Shapiro, M., et al. (2014). Maternal buffering of human amygdala-prefrontal circuitry during childhood but not during adolescence. Psychol Sci 25, 2067-2078. doi: 10.1177/0956797614550878 
Goursaud, A. P., and Bachevalier, J. (2007). Social attachment in juvenile monkeys with neonatal lesion of the hippocampus, amygdala and orbital frontal cortex. Behav. Brain Res. 176, 75-93. doi: 10.1016/j.bbr.2006.09.020

Graham, A. M., Buss, C., Rasmussen, J. M., Rudolph, M. D., Demeter, D. V., Gilmore, J. H., et al. (2016). Implications of newborn amygdala connectivity for fear and cognitive development at 6-months-of-age. Dev. Cogn. Neurosci. 18, 12-25. doi: 10.1016/j.dcn.2015.09.006

Gunnar, M. R., and Donzella, B. (2002). Social regulation of the cortisol levels in early human development. Psychoneuroendocrinology 27, 199-220. doi: 10.1016/s0306-4530(01)00045-2

Gunnar, M. R., Hostinar, C. E., Sanchez, M. M., Tottenham, N., and Sullivan, R. M. (2015). Parental buffering of fear and stress neurobiology: reviewing parallels across rodent, monkey and human models. Soc. Neurosci. 10, 474-478. doi: 10.1080/17470919.2015.1070198

Hanson, J. L., Nacewicz, B. M., Sutterer, M. J., Cayo, A. A., Schaefer, S. M., Rudolph, K. D., et al. (2015). Behavioral problems after early life stress: contributions of the hippocampus and amygdala. Biol. Psychiatry 77, 314-323. doi: 10.1016/j.biopsych.2014.04.020

Hanson, J. L., Williams, A. V., Bangasser, D. A., and Peña, C. J. (2021). Impact of early life stress on reward circuit function and regulation. Front. Psychiatry 12:744690. doi: 10.3389/fpsyt.2021.744690

Harlow, H., and Harlow, M. (1965). "The affectional systems," in Behavior of Nonhuman Primates, Vol. 2, eds A. Schrier, H. Harlow and F. Stollnitz (New York: Academic Press), 287-334.

Hennessy, M. B., Hornschuh, G., Kaiser, S., and Sachser, N. (2006). Cortisol responses and social buffering: a study throughout the life span. Horm. Behav. 49, 383-390. doi: 10.1016/j.yhbeh.2005.08.006

Hennessy, M. B., Kaiser, S., and Sachser, N. (2009). Social buffering of the stress response: diversity, mechanisms and functions. Front. Neuroendocrinol. 30, 470-482. doi: 10.1016/j.yfrne.2009.06.001

Hess, E. (1962). "Ethology: an approach to the complete analysis of behavior," in New Directions in Psychology, eds R. Brown, E. Galanter, E. Hess and G. Mendler (New York: Holt, Rinehart and Winston), 159-199.

Hornstein, E. A., and Eisenberger, N. I. (2017). Unpacking the buffering effect of social support figures: social support attenuates fear acquisition. PLoS One 12:e0175891. doi: 10.1371/journal.pone.0175891

Howell, B. R., McMurray, M. S., Guzman, D. B., Nair, G., Shi, Y., McCormack, K. M., et al. (2017). Maternal buffering beyond glucocorticoids: impact of early life stress on corticolimbic circuits that control infant responses to novelty. Soc. Neurosci. 12, 50-64. doi: 10.1080/17470919.2016.1200481

Hu, H., Cui, Y., and Yang, Y. (2020). Circuits and functions of the lateral habenula in health and in disease. Nat. Rev. Neurosci. 21, 277-295. doi: 10.1038/s41583020-0292-4

Huganir, R. L., and Nicoll, R. A. (2013). AMPARs and synaptic plasticity: the last 25 years. Neuron 80, 704-717. doi:10.1016/j.neuron.2013. 10.025

Inagaki, T. K., and Eisenberger, N. I. (2012). Neural correlates of giving support to a loved one. Psychosom. Med. 74, 3-7. doi: 10.1097/PSY.0b013e31823 59335

Jessen, S., and Grossmann, T. (2014). Unconscious discrimination of social cues from eye whites in infants. Proc. Natl. Acad. Sci. U S A 111, 16208-16213. doi: 10.1073/pnas.1411333111

Johansen, J. P., Cain, C. K., Ostroff, L. E., and LeDoux, J. E. (2011). Molecular mechanisms of fear learning and memory. Cell 147, 509-524. doi: 10.1016/j. cell.2011.10.009

Kehoe, P., Shoemaker, W., Arons, C., Triano, L., and Suresh, G. (1998). Repeated isolation stress in the neonatal rat: relation to brain dopamine systems in the 10-day-old rat. Behav. Neurosci. 112, 1466-1474. doi: 10.1037/0735-7044.112. 6.1466

Kennedy, D. P., and Adolphs, R. (2012). The social brain in psychiatric and neurological disorders. Trends Cogn. Sci. 16, 559-572. doi: 10.1016/j.tics.2012. 09.006

Kienast, T., Hariri, A. R., Schlagenhauf, F., Wrase, J., Sterzer, P., Buchholz, H. G., et al. (2008). Dopamine in amygdala gates limbic processing of aversive stimuli in humans. Nat. Neurosci. 11, 1381-1382. doi: 10.1038/nn.2222

Levine, S. (2001). Primary social relationships influence the development of the hypothalamic--pituitary--adrenal axis in the rat. Physiol. Behav. 73, 255-260. doi: 10.1016/s0031-9384(01)00496-6
Levine, S., Johnson, D. F., and Gonzalez, C. A. (1985). Behavioral and hormonal responses to separation in infant rhesus monkeys and mothers. Behav. Neurosci. 99, 399-410. doi: 10.1037//0735-7044.99.3.399

Levine, S., Stanton, M. E., and Gutierrez, Y. R. (1988). Maternal modulation of pituitary-adrenal activity during ontogeny. Adv. Exp. Med. Biol. 245, 295-310. doi: 10.1007/978-1-4899-2064-5_24

Lorétan, K., Bissière, S., and Lüthi, A. (2004). Dopaminergic modulation of spontaneous inhibitory network activity in the lateral amygdala. Neuropharmacology 47, 631-639. doi: 10.1016/j.neuropharm.2004.07.015

Malter Cohen, M., Jing, D., Yang, R. R., Tottenham, N., Lee, F. S., and Casey, B. J. (2013). Early-life stress has persistent effects on amygdala function and development in mice and humans. Proc. Natl. Acad. Sci. U S A 110, 18274-18278. doi: 10.1073/pnas.1310163110

Maren, S., and Fanselow, M. S. (1996). The amygdala and fear conditioning: has the nut been cracked? Neuron 16, 237-240. doi: 10.1016/s0896-6273(00) 80041-0

Masten, A. S., Burt, K. B., Roisman, G. I., Obradovic, J., Long, J. D., and Tellegen, A. (2004). Resources and resilience in the transition to adulthood: continuity and change. Dev. Psychopathol. 16, 1071-1094. doi: 10.1017/s095457940 4040143

Meyer, H. C., and Lee, F. S. (2019). Translating developmental Neuroscience to Understand risk for psychiatric disorders. Am. J. Psychiatry 176, 179-185. doi: 10.1176/appi.ajp.2019.19010091

Miller, A. (2007). Social neuroscience of child and adolescent depression. Brain Cogn. 65, 47-68. doi: 10.1016/j.bandc.2006.02.008

Moriceau, S., and Sullivan, R. M. (2006). Maternal presence serves as a switch between learning fear and attraction in infancy. Nat. Neurosci. 9, 1004-1006. doi: $10.1038 / \mathrm{nn} 1733$

Nachmias, M., Gunnar, M., Mangelsdorf, S., Parritz, R. H., and Buss, K. (1996). Behavioral inhibition and stress reactivity: the moderating role of attachment security. Child Dev. 67, 508-522. doi: 10.2307/1131829

Ng, K. H., Pollock, M. W., Urbanczyk, P. J., and Sangha, S. (2018). Altering D1 receptor activity in the basolateral amygdala impairs fear suppression during a safety cue. Neurobiol. Learn. Mem. 147, 26-34. doi: 10.1016/j.nlm. 2017.11.011

Oliver, C. F., Kabitzke, P., Serrano, P., Egan, L. J., Barr, G. A., Shair, H. N. et al. (2016). Repeated recall and $\mathrm{PKM} \zeta$ maintain fear memories in juvenile rats. Learn. Mem. 23, 710-713. 10.1101/lm.042549.116

Opendak, M., Gould, E., and Sullivan, R. (2017). Early life adversity during the infant sensitive period for attachment: programming of behavioral neurobiology of threat processing and social behavior. Dev. Cogn. Neurosci. 25 , 145-159. doi: 10.1016/j.dcn.2017.02.002

Opendak, M., Raineki, C., Perry, R. E., Rincon-Cortes, M., Song, S. C., Zanca, R. M., et al. (2021). Bidirectional control of infant rat social behavior via dopaminergic innervation of the basolateral amygdala. Neuron 109 , 4018-4035.e7. doi: 10.1016/j.neuron.2021.09.041

Opendak, M., Robinson-Drummer, P., Blomkvist, A., Zanca, R. M., Wood, K., Jacobs, L., et al. (2019). Neurobiology of maternal regulation of infant fear: the role of mesolimbic dopamine and its disruption by maltreatment. Neuropsychopharmacology 44, 1247-1257. doi: 10.1038/s41386-019-0340-9

Opendak, M., Theisen, E., Blomkvist, A., Hollis, K., Lind, T., Sarro, E., et al. (2020). Adverse caregiving in infancy blunts neural processing of the mother. Nat. Commun. 11:1119. doi: 10.1038/s41467-020-14801-3

Opendak, M., Zanca, R. M., Anane, E., Serrano, P. A., and Sullivan, R. M. (2018). Developmental transitions in amygdala $\mathrm{PKC}$ isoforms and AMPA receptor expression associated with threat memory in infant rats. Sci. Rep. 8:14679. doi: 10.1038/s41598-018-32762-y

Packard, K., Hu, S., Wang, J., and Opendak, M. (2021). Social regulation of developing mesolimbic circuitry. Society for Neuroscience, Virtual.

Patel, D., Kas, M. J., Chattarji, S., and Buwalda, B. (2019). Rodent models of social stress and neuronal plasticity: relevance to depressive-like disorders. Behav. Brain Res. 369:111900. doi: 10.1016/j.bbr.2019.111900

Perone, S., and Gartstein, M. A. (2019). Relations between dynamics of parentinfant interactions and baseline EEG functional connectivity. Infant. Behav. Dev. 57:101344.. doi: 10.1016/j.infbeh.2019.101344

Phelps, E. A., and LeDoux, J. E. (2005). Contributions of the amygdala to emotion processing: from animal models to human behavior. Neuron 48, 175-187. doi: 10.1016/j.neuron.2005.09.025 
Pratt, M., Zeev-Wolf, M., Goldstein, A., and Feldman, R. (2019). Exposure to early and persistent maternal depression impairs the neural basis of attachment in preadolescence. Prog. Neuropsychopharmacol. Biol. Psychiatry 93, 21-30. doi: 10.1016/j.pnpbp.2019.03.005

Proulx, C. D., Hikosaka, O., and Malinow, R. (2014). Reward processing by the lateral habenula in normal and depressive behaviors. Nat. Neurosci. 17, 1146-1152. doi: 10.1038/nn.3779

Raineki, C., Cortes, M. R., Belnoue, L., and Sullivan, R. M. (2012). Effects of early-life abuse differ across development: infant social behavior deficits are followed by adolescent depressive-like behaviors mediated by the amygdala. J. Neurosci. 32, 7758-7765. doi: 10.1523/JNEUROSCI.5843-11.2012

Raineki, C., Opendak, M., Sarro, E., Showler, A., Bui, K., McEwen, B. S., et al. (2019). During infant maltreatment, stress targets hippocampus, but stress with mother present targets amygdala and social behavior. Proc. Natl. Acad. Sci. U S A 116, 22821-22832. doi: 10.1073/pnas.1907170116

Raineki, C., Sarro, E., Rincon-Cortes, M., Perry, R., Boggs, J., Holman, C. J., et al. (2015). Paradoxical neurobehavioral rescue by memories of early-life abuse: the safety signal value of odors learned during abusive attachment. Neuropsychopharmacology 40, 906-914. doi: 10.1038/npp.2014.266

Raineki, C., Shionoya, K., Sander, K., and Sullivan, R. M. (2009). Ontogeny of odor-LiCl vs. odor-shock learning: similar behaviors but divergent ages of functional amygdala emergence. Learn. Mem. 16, 114-121. doi: 10.1101/lm. 977909

Raper, J., Stephens, S. B., Sanchez, M., Bachevalier, J., and Wallen, K. (2014). Neonatal amygdala lesions alter mother-infant interactions in rhesus monkeys living in a species-typical social environment. Dev. Psychobiol. 56, 1711-1722. doi: 10.1002/dev.21234

Robinson-Drummer, P. A., Opendak, M., Blomkvist, A., Chan, S., Tan, S., Delmer, C., et al. (2019). Infant trauma alters social buffering of threat learning: emerging role of prefrontal cortex in preadolescence. Front. Behav. Neurosci. 13:132. doi: 10.3389/fnbeh.2019.00132

Rosenkranz, J. A., and Grace, A. A. (2002). Dopamine-mediated modulation of odour-evoked amygdala potentials during pavlovian conditioning. Nature 417, 282-287. doi: 10.1038/417282a

Rudy, J. W., and Cheatle, M. D. (1983). Odor-aversion learning by rats following LiCl exposure: ontogenetic influences. Dev. Psychobiol. 16, 13-22. doi: 10.1002/dev.420160103

Sahi, R. S., Schwyck, M. E., Parkinson, C., and Eisenberger, N. I. (2021). Having more virtual interaction partners during COVID-19 physical distancing measures may benefit mental health. Sci. Rep. 11:18273. doi: 10.1038/s41598021-97421-1

Shultz, S., Klin, A., and Jones, W. (2018). Neonatal transitions in social behavior and their implications for autism. Trends Cogn. Sci. 22, 452-469. doi: 10.1016/j. tics.2018.02.012

Simmons, S. C., Shepard, R. D., Gouty, S., Langlois, L. D., Flerlage, W. J., Cox, B. M., et al. (2020). Early life stress dysregulates kappa opioid receptor signaling within the lateral habenula. Neurobiol. Stress 13:100267. doi: 10.1016/j.ynstr.2020.100267

Southwick, S. M., Bonanno, G. A., Masten, A. S., Panter-Brick, C., and Yehuda, R. (2014). Resilience definitions, theory and challenges: interdisciplinary perspectives. Eur. J. Psychotraumatol. 5:25338. doi: 10.3402/ejpt.v5. 25338

Stamoulis, C., Vanderwert, R. E., Zeanah, C. H., Fox, N. A., and Nelson, C. A. (2015). Early psychosocial neglect adversely impacts developmental trajectories of brain oscillations and their interactions. J. Cogn. Neurosci. 27, 2512-2528. doi: 10.1162/jocn_a_00877

Sullivan, R. M., and Holman, P. J. (2010). Transitions in sensitive period attachment learning in infancy: the role of corticosterone. Neurosci. Biobehav. Rev. 34, 835-844. doi: 10.1016/j.neubiorev.2009.11.010

Sullivan, R. M., Landers, M., Yeaman, B., and Wilson, D. A. (2000). Good memories of bad events in infancy. Nature 407, 38-39. doi: 10.1038/35024156
Sullivan, R. M., and Wilson, D. A. (1994). The locus coeruleus, norepinephrine and memory in newborns. Brain Res. Bull. 35, 467-472. doi: 10.1016/03619230(94)90160-0

Sullivan, R. M., Zyzak, D. R., Skierkowski, P., and Wilson, D. A. (1992). The role of olfactory bulb norepinephrine in early olfactory learning. Brain Res. Dev. Brain Res. 70, 279-282. doi: 10.1016/0165-3806(92)90207-d

Tamborski, A., Lucot, J. B., and Hennessy, M. B. (1990). Central dopamine turnover in guinea pig pups during separation from their mothers in a novel environment. Behav. Neurosci. 104, 607-611. doi: 10.1037//0735-7044.104.4. 607

Tedeschi, R. G., and Calhoun, L. G. (2004). Posttraumatic growth: conceptual foundations and empirical evidence. Psychol. Inquiry 15, 1-18. doi: 10.1207/s15327965pli1501_01

Teicher, M. H., Andersen, S. L., Polcari, A., Anderson, C. M., and Navalta, C. P. (2002). Developmental neurobiology of childhood stress and trauma. Psychiatr. Clin. North Am. 25, 397-426. doi: 10.1016/s0193-953x(01)00003-x

Teicher, M. H., Andersen, S. L., Polcari, A., Anderson, C. M., Navalta, C. P., and Kim, D. M. (2003). The neurobiological consequences of early stress and childhood maltreatment. Neurosci. Biobehav. Rev. 27, 33-44. doi: 10.1016/s0149-7634(03)00007-1

Thompson, J. V., Sullivan, R. M., and Wilson, D. A. (2008). Developmental emergence of fear learning corresponds with changes in amygdala synaptic plasticity. Brain Res. 1200, 58-65. doi: 10.1016/j.brainres.2008. 01.057

Tottenham, N. (2012). Human amygdala development in the absence of species-expected caregiving. Dev. Psychobiol. 54, 598-611. doi: 10.1002/dev. 20531

Tottenham, N. (2020). Early adversity and the neotenous human brain. Biol. Psychiatry 87, 350-358. doi: 10.1016/j.biopsych.2019.06.018

Tottenham, N., Shapiro, M., Flannery, J., Caldera, C., and Sullivan, R. M. (2019). Parental presence switches avoidance to attraction learning in children. Nat. Hum. Behav. 3, 1070-1077. doi: 10.1038/s41562-019-0656-9

Tye, K. M., Tye, L. D., Cone, J. J., Hekkelman, E. F., Janak, P. H., and Bonci, A. (2010). Methylphenidate facilitates learning-induced amygdala plasticity. Nat. Neurosci. 13, 475-481. doi: 10.1038/nn.2506

Upton, K. J., and Sullivan, R. M. (2010). Defining age limits of the sensitive period for attachment learning in rat pups. Dev. Psychobiol. 52, 453-464. doi: $10.1002 /$ dev.20448

VanTieghem, M. R., and Tottenham, N. (2018). Neurobiological programming of early life stress: functional development of amygdala-prefrontal circuitry and vulnerability for stress-related psychopathology. Curr. Top Behav. Neurosci. 38, 117-136. doi: 10.1007/7854_2016_42

Conflict of Interest: The authors declare that the research was conducted in the absence of any commercial or financial relationships that could be construed as a potential conflict of interest.

Publisher's Note: All claims expressed in this article are solely those of the authors and do not necessarily represent those of their affiliated organizations, or those of the publisher, the editors and the reviewers. Any product that may be evaluated in this article, or claim that may be made by its manufacturer, is not guaranteed or endorsed by the publisher.

Copyright (c) $2022 \mathrm{Hu}$, Packard and Opendak. This is an open-access article distributed under the terms of the Creative Commons Attribution License (CC BY). The use, distribution or reproduction in other forums is permitted, provided the original author(s) and the copyright owner(s) are credited and that the original publication in this journal is cited, in accordance with accepted academic practice. No use, distribution or reproduction is permitted which does not comply with these terms. 